\title{
Corrected transposition of the great arteries without associated defects in adult patients: clinical profile and follow up
}

\author{
Patrizia Presbitero, Jane Somerville, Filippo Rabajoli, Susan Stone, Maria Rosa Conte
}

\begin{abstract}
Objective-To assess the clinical course of adult patients with corrected transposition of the great arteries without associated anomalies.

Design-All patients with corrected
\end{abstract} transposition of the great arteries without associated anomalies were reviewed with complete clinical and echocardiographic assessment. The complications were evaluated in each decade.

Setting-Tertiary centre with a specific unit dealing with "grown-up" adolescent and adult congenital heart disease, designated as a quaternary centre and a general hospital with a referral centre for "grown-up" congenital heart disease.

Patients-18 patients (nine male and nine female) aged 16-61 years followed for 1-30 years (mean 10 years).

Results-There were no deaths. Six patients had a worsening ability index during follow up. Complications were: (a) complete heart block in seven, three of whom required pacemaker insertion; (b) significant left atrioventricular valve regurgitation in $50 \%$, appearing only in the third decade $(12 \%)$, with increasing frequency thereafter. Infective endocarditis was responsible for increasing left atrioventricular valve regurgitation in only one patient; (c) supraventricular arrhythmia appeared in the fifth decade, and occurred in all patients over the age of 60 years. One patient aged 61 had recurrent sustained ventricular tachycardia; and (d) congestive heart failure developed only after 50 years in $66 \%$. One patient had severe left atrioventricular valve regurgitation; the function of the systemic ventricle was only moderately reduced in the other three. Three of the nine women had seven uneventful pregnancies.

Conclusions-Patients with corrected transposition of the great arteries without associated defects may remain undiagnosed until adult life. Symptoms occur rarely before the fourth and fifth decades, when rhythm disturbance, left atrioventricular valve regurgitation, and moderately impaired systemic ventricular function cause congestive cardiac failure. The role of pacemaker insertion or surgery for left atrioventricular valve regurgitation needs further assessment.

(Br Heart f 1995;74:57-59)
Keywords: corrected transposition of the great arteries without associated defects; natural history of corrected transposition of the great arteries; "grown-up" congenital heart disease.

Corrected transposition of the great arteries (atrioventricular discordance and ventriculoarterial discordance) as a lone anomaly is rare. Patients with this condition reach adult life without earlier diagnosis or recognition of having heart disease. Left atrioventricular valve regurgitation, complete heart block, and systemic ventricular dysfunction develop as the years advance. The incidence of such complications and the age at which they occur have not been completely documented. The aim of this survey was to illustrate the natural history of a group of patients with corrected transposition of the great arteries without associated defects.

\section{Patients and methods}

Data were collected from two centres specialising in the care of patients with "grown up" congenital heart disease, namely, the Royal Brompton National Heart and Lung Hospital (RBH), London, United Kingdom, and the Hospital Giovanni Bosco (HGB), Turin, Italy. From this population data of patients with corrected transposition of the great arteries followed in adulthood were reviewed retrospectively.

Selection criteria were: (a) age of patients 16 years or over at first presentation in the centre; (b) follow up of more than 1 year; and (c) diagnosis of corrected transposition of the great arteries without major associated defects confirmed by echocardiography or angiography, or both.

There were 18 patients ( 10 from the $R B H$ and eight from the HGB). The mean (range) age at presentation was $35(16-61)$ years and mean (range) follow up of the 18 patients was $10(1-30)$ years. All patients were reviewed with complete clinical and echocardiographic assessment. The ability index (AI) ${ }^{1}$ was used for grading symptoms. The complications, evaluated decade by decade, were incidence of arrhythmia, complete heart block, cardiac failure, left atrioventricular valve regurgitation, and bacterial endocarditis.

\section{Results}

The reasons for the initial referral of these patients to a cardiologist were (a) abnormal electrocardiogram ( $Q$ wave in V1-V3) in three patients, one of which was interpreted 


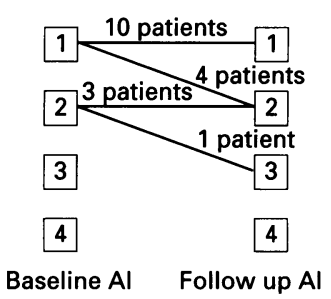

Figure 1 Ability index at baseline and at the end of follow up (mean 10 years). $A I$, ability index. for years as an anterior myocardial infarction; (b) complete heart block in four, three of whom probably had it from birth; (c) abnormal findings in routine chest $x$ ray film in two; (d) discovery of a systolic murmur due to mild left atrioventricular valve regurgitation in five; (e) paroxysmal atrial arrhythmia in three; and (f) questionable cyanosis in a patient who presented with a systolic murmur. Cyanosis was related to a left superior vena cava entering the left atrium.

When first seen 14 of 18 patients were classified as $\mathrm{AI} 1$-that is well and asymptomatic (fig 1) and four were AI 2. After a mean (range) of $10(1-30)$ years 10 patients were still AI 1, seven were symptomatic AI 2, and one was AI 3 from an hemiplegia related to atrial fibrillation. There were no deaths.

Figure 2 shows the prevalence of complications for each decade. Complete atrioventricular block was congenital in three patients $(17 \%)$ of the population and was acquired in further four patients $(22 \%)$ at ages $15,30,35$, and 44 years. The percentage of patients with complete atrioventricular block, congenital or acquired, in each decade was stable between $25 \%$ and $33 \%$; only three patients (two with congenital and one with acquired complete atrioventricular block) had insertion of a pacemaker because of a slow deterioration in their clinical state (dizziness and low ventricular rate) at the age of 28,35 , and 36 years. The other four patients remain asymptomatic with good heart rate response during effort up to ages $22,26,41$, and 61 years.

Significant left atrioventricular valve regurgitation appeared only in the third decade in $12 \%$ and became progressively severe in the following decades; it was established in four of six patients who reached the sixth decade. Surgical intervention was considered necessary for severe valvular regurgitation in one patient aged 58 years but was not performed because of serious deterioration from a hemiparesis from embolism with atrial fibrillation. Left atrioventricular valve regurgitation was mild in five patients and moderate in three. The left atrioventricular valve was mildly malformed in only three patients. No patient had left sided Ebstein's anomaly.

Supraventricular arrhythmia began to appear in the fifth decade in three of six patients, but affected five of the six who

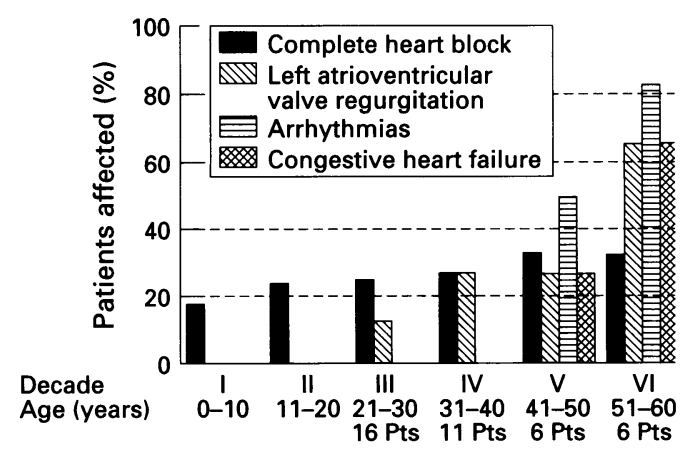

Figure 2 Prevalence of complications for each decade. (Pts, patients.)

(1th, patients. reached 51-60 years (two with atrial fibrillation and three with paroxysmal supraventricular tachycardia). One patient, a 61 year old woman with good ventricular function, developed sustained ventricular tachycardia.

Congestive heart failure appeared for the first time in the fifth and sixth decades. One patient acquired severe left atrioventricular valve regurgitation and three had moderate regurgitation. All of this group had impairment of systemic ventricular function, two with additional complete heart block. In one patient cardiac failure developed 2 months after an attack of bacterial endocarditis on the left atrioventricular valve which did not cause severe regurgitation at the time but which became more important 2 years later.

Three patients had uneventful pregnancies, with seven live born infants, none of whom had congenital heart disease.

\section{Discussion}

Additional structural cardiac anomalies usually coexist with the malformation of corrected transposition of the great arteries, ${ }^{2}$ but when not present the natural history would seem from this series to be good at least until the fifth decade of life. Whether such patients die in infancy and childhood is not known and not mentioned in reported series of corrected transposition. Obviously heart block leading to sudden death or requiring pacemaker insertion can occur at any time even in utero with and without associated anomalies. The progressive deterioration with the onset of cardiac failure after 40 years and certainly 50 years means that life expectancy is not normal. This deterioration comes from several factors which may appear together; complete heart block, increasing left atrioventricular valve regurgitation closely associated with deterioration in systemic ventricular performance, increasing left atrial pressure, and predisposing to atrial flutter and fibrillation. These factors herald rapid deterioration and changing ability indices. Most patients require medical treatment by $45-50$ years - that is, diuretics, angiotensin converting enzyme (ACE) inhibitors, and antiarrhythmic drugs. The decision whether or not to operate on the left atrioventricular valve is difficult, because it is hard to differentiate the role of ventricular dysfunction from regurgitation of the valve, which is usually anatomically abnormal.

From the literature, ${ }^{3-7} 16$ patients over 40 years of age (range $42-80$ years) without associated anomalies have been reported; 10 of these had mild/moderate left atrioventricular valve regurgitation and more than one third of the cases had an episode of heart failure after the fifth decade.

The function of the systemic ventricle in corrected transposition has been debated. Most authors recognise the functional vulnerability of the morphological right ventricle in the systemic location with ageing. ${ }^{7}$ We agree, however, with Dimas et ap who found only mild impairment of morphological right ventricular function in 18 adults even with 
associated anomalies in the majority. In three of four patients with overt heart failure, the systemic ventricle was only mildly impaired. This is only one of the factors, and perhaps not the most important, which contribute to the development of heart failure.

There are few reports about the function of the right sided ventricle. We do not have any evidence to suggest that there is any dysfunction with increasing age. If pulmonary stenosis is present, however, right sided dysfunction may develop insidiously.

The left atrioventricular valve tends to become incompetent with time in patients with corrected transposition of the great arteries without associated anomalies appearing in adult life. It is difficult to differentiate the role of systemic ventricular dilatation, together with tricuspid ring enlargement, from acquired deformity of the cusp in causing tricuspid regurgitation-present in $30 \%$ after 40 years of age. Certainly in our series we did not have evidence of the presence of Ebstein's malformation but we could not exclude the presence of a milder degree of this abnormality. Even if none of our patients had left atrioventricular valve replacement, at the present time surgery should be considered when regurgitation is moderate or severe and while the left sided ventricular function is still preserved. In the future, through transoesophageal echocardiography, we may be able to understand more precisely the mechanism of atrioventricular valve regurgitation and may be able to intervene earlier.

Patients with corrected transposition of the great arteries without associated anomalies should be protected from bacterial endocarditis. Infection occurs on the left atrioventricular valve despite apparent normal function. In one patient left atrioventricular valve regurgitation progressed to become severe within 2 years of an episode of endocarditis.

It is our practice to introduce ACE inhibitors with the appearance of mild regurgitation and the first sign of reduced ventricular function in the hope of slowing or halting deterioration even if we do not have any data to support the evidence. It is therefore important to follow patients with good echocardiographic assessment of ventricular and atrioventricular valve function. Transoesophageal echocardiography gives much better assessment than transthoracic echocardiography in these patients with grown up congenital heart disease and should be used in routine supervision with biannual or annual evaluation if clinical changes occur.

Supraventricular arrhythmia occurred in five of the six patients in the sixth decade, causing pulmonary oedema in some. Drugs with a negative inotropic effect should not be used. In patients with atrial fibrillation or flutter anticoagulants must be used early because of the embolic risks and cardioversion attempted early. Our policy is to give amiodarone, ACE inhibitors, and diuretics with careful monitoring of potassium concentrations in all patients after onset of the first attack, however short lived. In this population ventricular arrhythmias were exceptional despite the presence of impaired systemic ventricular function in several patients. Atrioventricular conduction disturbances are common, with a high percentage of established complete heart block occurring at different ages, starting in any decade, but always with warning symptoms. No sudden, unexpected deaths occurred in this group. Patients with corrected transposition of the great arteries are warned to report to the centre immediately if symptoms of presyncope, syncope, or a sudden increase in fatigue or dyspnoea occur. Pacemaker insertion can be delayed until symptoms or chronotrophic regurgitation occur. The ventricular rate response during effort is often good and the development of more advanced conduction disturbance or slow ventricular rates tend to be gradual. The use of the dual chamber pacemaker is mandatory considering the age of the patients ${ }^{8}$ and the constant progressive deterioration of systemic ventricular function. The effect of earlier pacemaker implantation to prevent the onset of atrial arrhythmia needs to be assessed.

Pregnancy was well tolerated and the percentage of live born infants without congenital anomalies was high.

This group of patients seems to have a good prognosis in the first three to four decades of life and this has important implications for insurance companies, driving licensing authorities, and employment bodies. They do, however, need careful follow up by a cardiologist who is able to recognise the first signs of the frequent complications which will occur after the fifth decade.

1 Warnes C, Somerville J. Tricuspid atresia in adolescents and adults: current state and late complications. $B r$ Heart f 1986;56:535-43.

2 Van Praagh R. What is congenitally corrected transposition? N Engl f Med 1970;282:1097-8.

3 Pezard P, Banus Y, Laporte J, Geslin P, Garnier H, Tade A. Transposition corrigèe des gros vaisseaux de l'adulte A. Transposition corrigee des gros vais
age. Arch Mal Coeur 1986;11:1637-42.

4 Horis H, Demoulin JC. La transposition corrigée des gros vaisseaux chez le sujet agé. Arch Mal Coeur 1981;8: 951-60.

5 Dimas AP, Moodie DS, Sterba R, Gill CC. Long-term function of the morphologic right ventricle in adult patients with corrected transposition of the great arteries. Am Heart f 1989;118:526-30.

6 Ikeda U, Kimura K, Suzuki O, Furuse M, Natsume T Long-term survival in corrected transposition. Lancet 1991;337:180-1.

7 Graham TP, Parrish MD, Boucek RJ, et al. Assessment of ventricular size and function in congenitally corrected ventricular size and function in congenitally corrected transposition

8 Marcolongo M, Presbitero P, Capra G, Chiotti D, Gobbi G. Bicameral stimulation in a case of corrected transposition of the great arteries. G Ital Cardiol 1986;16 798-802. 